\title{
In Vivo Visualization of Collagen Transdermal Absorption by a Combined Second-harmonic Generation and Two-photon Excited Fluorescence Method
}

\section{yanan Sun}

China Academy of Chinese Medical Sciences

\section{Shuhua Ma}

China Academy of Chinese Medical Sciences

Gaiying He

China Academy of Chinese Medical Sciences

\section{Weifeng Yang}

China Academy of Chinese Medical Sciences

Yi Wang ( $\nabla$ prof.wangyi@foxmail.com )

China Academy of Chinese Medical Sciences

\section{Research Article}

Keywords: Transdermal absorption, Second-harmonic generation, Two-photon excited fluorescence, Recombinant human collagen

Posted Date: August 31st, 2021

DOl: https://doi.org/10.21203/rs.3.rs-801514/v1

License: (c) (i) This work is licensed under a Creative Commons Attribution 4.0 International License. Read Full License 


\section{Abstract}

The transdermal administration of collagen is an important method used for wound healing and skin regeneration. However, due to the limitations of previous approaches, the process and degree of collagen transdermal absorption could only be quantitatively and qualitatively assessed in vitro. In the present study, we introduced a novel approach combining second-harmonic generation with two-photon excited fluorescence to visualize the dynamics of collagen transdermal absorption in vivo. The high resolution images showed that the exogenous recombinant human collagen permeated through the epidermis, reached the dermis and formed reticular structures in real time. We also validated these findings through traditional in vitro skin scanning and histological examination. Thus, our approach provides a reliable method of measurement for the real-time evaluation of collagen absorption and treatment effects.

\section{Introduction}

The skin is the largest organ in the human body and plays a key role in protecting our bodies from the varied external environment [1]. It is mainly composed of three layers that span from superficial to deep, including the epidermis, dermis, and hypodermis [2]. The dermal skin contains components of the extracellular matrix, such as collagen fibers, that contribute to the strength and toughness of the skin[3]. As the main component of connective tissue, collagen is the most abundant protein in skin, comprising $25 \%$ to $35 \%$ of the protein content in the whole body [4]. Collagen is also widely used in cosmetics and biomedical products $[5,6]$. After exogenous collagen is smeared on the skin, it permeates through the cuticle to the dermis and forms a reticular structure to support the structure of the dermis and supply more extracellular matrix to keep the skin elastic [7]. Thus, we need to dynamically visualize the process of the transdermal absorption of exogenous collagen and measure the extent of collagen absorption with qualitative and quantitative approaches.

To date, various technologies have been applied to measure the biomedical administration of collagen on skin, including chemical assays $\left[{ }^{8}\right]$, tissue inspection [9], isotope tracing [10] and circulating diffusion chamber [11], which can only be used to quantitatively examine the transdermal absorption of exogenous collagen in vitro. However, these approaches could not dynamically track the process of transdermal absorption in vivo and evaluate the real-time effects of biomedical administration. These limitations could be overcome by nonlinear optical microscopy, such as two-photon excitation fluorescence (TPEF) $[12,13]$, and second harmonic generation (SHG) [14, 15]]]]] []]] \{Campagnola, 2003 \#14;Thrasivoulou, 2011 \#17;Pittet, $2014 \# 16\}]$. Among such technologies, SHG is a well-established method to directly visualize anisotropic biological tissues that possess large hyperpolarizabilities, such as collagen[16]. Moreover, SHG imaging has many advantages, such as a higher signal-to-noise ratio than autofluorescence imaging, the detection intrinsic signals without extrinsic dyes and the utilization of nonlinear excitation from molecular fluorescence[17]. Nonetheless, few studies have examined the effects of drug administration on the skin using SHG because the challenges in using it to distinguish endogenous from exogenous collagen. Recently, two-photon excited fluorescence (TPEF) imaging has been widely applied to visualize the dynamics of intrinsic molecules and cells in living specimens through 
conventional labeling via staining or fluorescent proteins [18]. Due to the divergent mechanisms of SHG and TPEF, we speculate that they could be combined to provide complementary information for the dynamic observation of biological processes. However, few studies have reported the in vivo imaging of the transdermal absorption of collagen with a combination of SHG and TPEF (SHG-TPEF). Therefore, there is an urgent need to obtain in vivo high-resolution images of fibrillar collagen during the process of transdermal absorption using SHG-TPEF imaging, which could provide a novel approach for estimating the extent of drug absorption and further assessing the treatment effects of dermal drugs.

To address these issues, we first constructed the mouse model of transdermal absorption through administrating exogenous recombinant human collagen (R-hc) which was proved to be effective in the skin absorption in our previous studies[19]. Second, we set up the platform of in vivo SHG-TPEF skin imaging. Third, we tracked the dynamic process of collagen absorption of short-term administration by in vivo SHG-TPEF imaging. Finally, we applied Franz-type diffusion method to evaluate the collagen absorption at different time points as validations.

\section{Experimental}

Animals

Healthy BALB/c mice were purchased from Vital River Laboratories (China). All the animals used in this study were male adults (6-8 weeks, $20 \pm 2 \mathrm{~g}$ body weight). Mice were kept in a specific pathogen-free environment with freely available food and water and a $12 \mathrm{~h}$ light-dark cycle. In this study, five mice were used at each time point, each of which received a topical dose of $1 \mathrm{mg} / \mathrm{ml}$ of R-hc on a shaved back on the left side of the thoracic spine and a saline control area on the right. The animal experiments were supervised and approved by the Research Ethics Committee of the China Academy of Chinese Medical Sciences( ERCCACMS21-2106-12).

\section{Quality control of R-hc}

$R$-hc: $\mathrm{R}$-hc is expressed by eukaryotic cells and integrates the human-like collagen gene, which is a heterozygous type I and type III collagen gene, into the yeast chromosome. The hydroxyproline in R-hc was replaced by proline. In our previous study, we analyzed the effect of R-hc on the repair of laser skin damage in mice. The results showed that R-hc can significantly accelerate wound healing, shorten wound healing time and promote collagen production in wounds[19], which suggests that R-hc could be transdermally absorbed.

Fluorescent label: For fluorescent labeling, a protein stock solution was prepared by mixing $100 \mathrm{mg}$ R-hc freeze-dried powder (TianJin Irheaya Biological Technology Co., Ltd., Tianjin) with $10 \mathrm{~mL} 1 \mathrm{M}$ phosphate buffer ( $\mathrm{pH}$ 8.5 9.0) to produce the $1 \mathrm{~mL}$ protein labeling stock solution. To prepare the dye stock solution, anhydrous DMSO was added into a vial of iFluor ${ }^{\text {TM }} 750$ SE (AAT Bioquest, Ex/Em=749/775 nm) to produce a 10-20 mM stock solution, which was mixed well by vortexing. Then, $1 \mathrm{~mL}$ of the dye stock solution was added to the vial of protein solution $(10 \mathrm{ml})$ and shaken thoroughly. The concentration of 
the protein was $10 \mathrm{mg} / \mathrm{ml}$. The reaction mixture was rotated at $4^{\circ} \mathrm{C}$ for $12 \mathrm{~h}$. The reaction mixture was loaded into a dialysis bag $(<1 \mathrm{kd})$ and placed in a large beaker containing ultra-pure water in the dark. The water was changed every $4 \mathrm{~h}$ until no fluorescence was detected in the ultra-pure water. The fluorescent protein was freeze-dried into a powder for future use.

\section{Immunofluorescence staining}

The localization of collagen III and collagen I protein was assessed by immunofluorescence. The skin tissue was taken from the left side of the thoracic spine after treated with R-hc for $0 \mathrm{~h}, 1 \mathrm{~h}, 2 \mathrm{~h}, 3 \mathrm{~h}, 4 \mathrm{~h}, 5$ $\mathrm{h}$ and $24 \mathrm{~h}$. The frozen sections were obtained from an ultralow temperature freezer after $30 \mathrm{~min}$ at room temperature. The sections were washed in $0.02 \mathrm{M}$ PBS 3 times and then blocked with QuickBlock ${ }^{\mathrm{TM}}$ Blocking Buffer for Immunological Staining (Beyotime, China) for $1 \mathrm{~h}$. A 1:500 dilution of anti-collagen III mouse monoclonal antibody and anti- collagen I rabbit polyclonal antibody (ab6310, ab34710; Abcam, USA) was added and incubated overnight at $4^{\circ} \mathrm{C}$. After washing with PBS, the sections were incubated for $1 \mathrm{~h}$ at room temperature in the dark with a secondary antibody (Dylight 488-goat-anti-mouse and Dylight 594-goat-anti-rabbit; EARTHox, China). After immunostaining, the specimens were examined with a widefield fluorescence microscope (BX51, Olympus, Tokyo, Japan) fitted with a digital camera (DP71; Olympus). The relative intensity of the staining was analyzed by Image Pro Plus 6.0 software (Media Cybernetics, Inc., USA).

\section{In vivo SHG-TPEF skin imaging}

The back hair was shaved from the mice, which were treated with iFluor-R-hc $(1 \mathrm{mg} / \mathrm{ml})$ for $0 \mathrm{~h}, 1 \mathrm{~h}, 2 \mathrm{~h}, 3$ $\mathrm{h}, 4 \mathrm{~h}$ and $5 \mathrm{~h}$ at room temperature while avoiding light. At the end of the experiment, all mice were anesthetized with Isoflurane and fixed on a mouse plate while the back skin was fixed with holder. The full-thickness skin tissue was scanned by a two-photon scanning confocal microscope (FV1000-MPE, Olympus, Japan).

A two-photon excited fluorescence microscopy system was used for image acquisition based on the reverse detection of SHG and two-photon excited fluorescence signals from the skin. The two-photon excited fluorescence microscopy system was equipped with a water-immersion objective lens (PLAN 25X OB. W IMM, NA 1.05, Olympus) and a Ti:sapphire laser oscillator (wavelength: 690-1040 nm; repetition rate: $80 \mathrm{MHz}$, pulse width: 100 fs; MaiTai HP DS-OL, Spectra-Physics, Inc. CA). The SHG image acquisition was performed at an excitation wavelength of $950 \mathrm{~nm}$ with a $690 \mathrm{~nm}$ dichroic mirror and a $475 / 20 \mathrm{~nm}$ emission filter (center wavelength/bandwidth). For the observation of iFluor-R-hc, an excitation wavelength of $790 \mathrm{~nm}$ and a $605 \mathrm{~nm}-680 \mathrm{~nm}$ emission filter were used to isolate the SHG signal. The autofluorescence image was acquired with an excitation wavelength of $750 \mathrm{~nm}$ and a 495 $\mathrm{nm}-540 \mathrm{~nm}$ emission filter.

The images were obtained from the epidermis and the dermis and stored as a z-stack image sequence (step size of $1 \mu \mathrm{m}$ in the $z$-axis). The image size was $512 \times 512$ pixels, and the scanning speed was 2 $\mu \mathrm{m} /$ pixel. The merging of the color channels was performed using FV1000 Viewer 3.1 software 
(Olympus). The three-dimensional image was performed using Imaris (Imaris ver. 8.4.2, Bitplane AG, Switzerland). To examine the collagen staining intensity, we calculated the SHG signal intensity, which has been considered a good predictor of collagen structure $[20,21]$. Thus, we calculated the signal intensity of SHG in the dermis at different time points after iFluor-R-hc administration and determined the collagen thickness based on the z-stack depth of the SHG signal.

\section{In vitro SHG-TPEF skin imaging}

Whole skin tissue $\left(1 \times 0.5 \mathrm{~cm}^{2}\right)$ was removed at $0 \mathrm{~h}, 1 \mathrm{~h}, 2 \mathrm{~h}, 3 \mathrm{~h}, 4 \mathrm{~h}$ and $5 \mathrm{~h}$ after the imaging of the mice. The tissues were examined as frozen sections $(10 \mu \mathrm{m})$. The lateral plane of the skin was exposed under the objective lens of a two-photon excited fluorescence microscopy system. The above mentioned scanning process was repeated to observe the iFluor-R-hc transdermal absorption. The laser condition settings were consistent with those used for the above-mentioned in vivo SHG-TPEF skin imaging.

\section{Hematoxylin and eosin staining}

All frozen sections were subjected to hematoxylin and eosin (HE) staining for the histological study. Differential interference contrast images of the sections were captured using an Olympus BX51 microscope (PlanApo 20x/NA: 0.45).

\section{The Franz-Type Diffusion}

Mouse skin was mounted in the receptor compartment of a Franz-type diffusion cell (TP-6, HT, Jintao Instrument Technology Co., Ltd., Tianjin, China). Ten milligrams of iFluor-R-hc dissolved in normal saline or normal saline alone was added to the tight interface facing the mouse skin. The donor cap was covered with tin foil. A $0.9 \%$ sodium chloride buffer solution $(\mathrm{pH} 7.4)$ was used as the receptor solution. The receptor solution was stirred by a magnetic stirrer rotating at $300 \mathrm{rpm}$ at $37^{\circ} \mathrm{C}$. The samples were examined at $1 \mathrm{~h}, 2 \mathrm{~h}, 3 \mathrm{~h}, 4 \mathrm{~h}$ and $6 \mathrm{~h}$. The amount of total protein in the receptor solution was measured by a BCA protein assay kit (Beyotime, China), and the fluorescence intensity was measured with a fluorescence microplate reader(Synergy H1Hybrid Multi-Mode Reader, BioTek Instruments, Inc., USA) at $\mathrm{Ex} / \mathrm{Em}=640 / 670 \mathrm{~nm}$.

\section{Gel electrophoresis}

The amount of total protein in the receptor solution was separated by sodium dodecyl sulfatepolyacrylamide gel electrophoresis (SDS-PAGE). The samples were stained with Coomassie Brilliant Blue R-250 stain (P0017F, Beyotime Biotechnology, China) then scanned with ChemiDoc ${ }^{\text {TM }}$ Touch Imaging System (Bio-Rad Laboratories, Inc., USA) .

\section{Statistical analysis}

The results were statistically analyzed by one-way ANOVA and differences between groups identified using Tukey's post-hoc test using GraphPad Prism v.6.0 (GraphPad software, Inc., La Jolla, Calif.). The 
difference was considered statistically significant at $p<0.05$. Post-hoc analysis was performed with $p<0.05$ as the threshold for statistical significance.

\section{Results}

R-hc transdermal absorption

After $24 \mathrm{~h}$ of R-hc administration, we observed that the fluorescence intensity of type III and type I collagen were significantly increased in the dermis relative to that observed in the control group (Fig. 1A). At the same time, we calculated the ratio of type III and type I collagen, compare to the control group the rate of increase of type III collagen was more notable. We also observed that type III collagen was interwoven into the reticular structures in the superficial dermis (Fig. 1B). The long fiber content of type III collagen in the R-hc treatment group was significantly increased compared with that in the control group (Fig. 1C). These findings suggest that R-hc could be transdermally absorbed and supplement type III and type I collagen in the dermis.

SHG-TPEF skin imaging

We characterized the structure of the skin and the iFluor-R-hc distribution from the epidermis to the dermis. Specifically, in the epidermis, we used TPEF imaging at an excitation wavelength of $750 \mathrm{~nm}$ to observe the stratum spinosum and stratum basale (Fig. 2A). Then, in the dermis, we separately used SHG and TPEF imaging at excitation wavelengths of $950 \mathrm{~nm}$ and $790 \mathrm{~nm}$, respectively, to visualize the dynamic process of iFluor-R-hc absorption (Fig. 2B ). Here, we used two-dimensional (2D) images and three-dimensional (3D) images to illustrate the scanning results. In the 2D images, we scanned the histological structures of the skin by utilizing the SHG signal $(\lambda=950 \mathrm{~nm})$ and iFluor-R-hc by utilizing the TPEF signal ( $\lambda=790 \mathrm{~nm}$ ) (Fig. 2B). Moreover, as shown in Fig. 2B, we revealed intrinsic collagen, and the exogenous ifluor-R-hc was mutually linked into reticular structures in the dermis.

Tracking the transdermal absorption of iFluor-R-hc using SHG-TPEF imaging in vivo

In this paper, the infrared fluorescent probe is used to label the collagen to be detected, which can avoid the autofluorescence of the skin, reducing background noise[22]. We first analyzed the mouse skin at the 0 $\mathrm{h}$ time point and showed a small amount of noise in the epidermis and the dermis and TPE autofluorescence in the hair shaft (Fig. 3A), which were considered to represent the baseline. Over time, we observed that the TPEF signal gradually increased (Fig. 3A), which suggests that iFluor-R-hc permeated through the cuticle and reached the dermis. Then, it formed reticular structures in the epidermis after $4 \mathrm{~h}$ (Fig. 3A). Statistically, the iFluor intensity significantly increased after $2 \mathrm{~h}$ administration of iFluor-R-hc (Fig. 3B), which suggests that R-hc was gradually absorbed after $2 \mathrm{~h}$ of application. Moreover, compared with the baseline, both the SHG signal intensity and collagen thickness were significantly increased after $4 \mathrm{~h}$ and $5 \mathrm{~h}$ of administration of iFluor-R-hc (Fig. 3C and 3D, $4 \mathrm{~h}$ time point: $t=5.43, p<0.001 ; 5 \mathrm{~h}$ time point: $t=4.68, p<0.001)$, which indicates the increased collagen density in the dermis. 
In addition, we found that iFluor-R-hc could permeate and disperse around hair follicles after $3 \mathrm{~h}$ of administration (Fig. 4). This result is consistent with the results of our previous in vitro experiments that the protein first accumulated in the hair follicle and then absorbed into the dermis [23], which suggests that R-hc may be absorbed into the dermis through the hair follicle.

To test whether our findings were the result of false positive signals in the in vivo SHG-TPEF imaging, we performed histological analysis. The results revealed that there is an increase tendency of HE staining but no statistical difference between each group(Fig. 5A). However, we found that both iFluor and the SHG intensity of iFluor-R-hc were significantly increased after $4 \mathrm{~h}$ and $5 \mathrm{~h}$ of administration of iFluor-R-hc (Fig. 5B and $5 \mathrm{C}$ ), which was consistent with the findings obtained from the in vivo SHG-TPEF imaging.

We used immunohistochemical methods to distinguish the changes of type I collagen and type III collagen with the time of R-hc administration in dermis. The results showed that the fluorescence intensity of type III collagen in the dermis began to increase significantly after the $4 \mathrm{~h}$ of R-hc administration, and the type I collagen increased significantly after 5 hours(Fig $6 \mathrm{~A}, \mathrm{~B}$ and $\mathrm{C}$ ). The ratio of type III collagen to type I collagen increased significantly after $2 \mathrm{~h}$ of R-hc administration(Fig 6D), suggesting an increase in the content of type III collagen in the dermis and it grows with the time of administration of R-hc.

In vitro tracking of the transdermal absorption of iFluor-R-hc

Next, we used traditional in vitro Franz diffusion cells to test whether in vivo SHG-TPEF imaging could sensitively and accurately track transdermal absorption, which could confirm the generality of our novel approach. Specifically, compared with the baseline $(0 \mathrm{~h})$, we first observed the significantly increased fluorescence intensity of iFluor-R-hc after $3 \mathrm{~h}$ of administration (Fig. 7A). Moreover, the iFluor-R-hc group showed significantly increased protein concentrations after $2 \mathrm{~h}$ of administration of iFluor-R-hc (Fig. 7B). Compared with the control group, the iFluor-R-hc group showed both significantly increased iFluor intensity and iFluor-R-hc concentration after $4 \mathrm{~h}$ of administration of iFluor-R-hc (Fig. 7C and 7D). These findings were consistent with the observations of the in vivo SHG-TPEF imaging, which suggests that our novel approach could sensitively and dynamically visualize collagen transdermal absorption in vivo. In addition, in the collected liquid, we found a clear target protein band about $55 \mathrm{kD}$ after $4 \mathrm{~h}$ of administration in the iFluor-R-hc group (Fig.7E), which suggests that R-hc can pentrate the full layer of skin after $4 \mathrm{~h}$ administration of iFluor-R-hc.

\section{Discussion}

In this study, we used a novel noninvasive approach to dynamically capture the process of collagen transdermal absorption in vivo by combining SHG with TPEF. Both two-photon fluorescence imaging and second harmonic imaging are second-order nonlinear processes. In addition to the needs of different filters addition, TPEF and SHG are almost identical on the optical detection system, the obtained information for both complement and confirm each other, so it has received much attention in recent years. In this paper, the infrared fluorescent probe is used to label the collagen, which can avoid the 
autofluorescence of the skin and reducing background noise [22]. Over time, we observed that collagen permeated through the hair follicle, reached the dermis and formed reticular structures in the dermis after $4 \mathrm{~h}$ of administration of exogenous iFluor-R-hc, which was also confirmed by the in vitro examinations. To the best of our knowledge, this approach is the first to be used for the in vivo visualization of the transdermal absorption of biochemically administered collagen, and these findings provide a technological method for the clinical assessment of transdermal drugs in the future.

A previous study showed that compounds with a molecular weight greater than $50 \mathrm{kDa}$ penetrate the dermis with difficulty because of blockage by the stratum corneum [24] , but whether macromolecules can penetrate the skin is still not clear. In our study, we used in vivo SHG-TPEF skin imaging to track the process of the transdermal absorption of exogenous collagen, which is approximately $55 \mathrm{kDa}$ in size. The iFluor fluorescence signal was first detected in the hair follicle $3 \mathrm{~h}$ after coating $\mathrm{R}$-hc on the skin, which confirms that macromolecules could be absorbed by the skin. Moreover, this combined technology has many advantages, such as noninvasiveness, the absence of conventional dyes and good penetration of biological tissues[17]. Thus, it allows the long-term observation of dynamic changes at the same skin site, which can be extended to the development of an in vivo real-time assessment method for cosmetics and biomedical administration.

The results of the examination of R-hc transdermal absorption provided a quantitative approach for the in vivo assessment of the transdermal absorption of protein drugs through the combined measurement of collagen density and absorption. Specifically, we first demonstrated that a typical application of R-hc increased type III collagen and collagen fiber growth, which suggests that R-hc could increase skin elasticity. Second, we used TPEF combined with SHG imaging to study the manner and depth of R-hc absorption in vivo. Since the stratum corneum acts as a physical barrier in skin on the surface of the epidermis [25], it has an impact on the penetration efficiency of R-hc. However, the results of the in vivo transdermal absorption model showed that the fluorescence intensity of type III collagen was significantly increased after $24 \mathrm{~h}$ of R-hc administration, which suggests that iFluor-R-hc could still effectively permeate the epidermal skin. Potts and colleagues found that an increased molecular weight increased lipophilicity and permeability, which suggests that the penetration of drugs into the skin is tightly associated with the molecular characteristics of the drug [26]. Lin and colleagues also considered that the effect of lipophilicity is a more dominant factor than the molecular weight in the passive diffusion permeability [27]. We observed the fluorescence signal of iFluor in the hair follicle after $3 \mathrm{~h}$ of Rhc application by in vivo SHG-TPEF imaging, and the dermis was interlaced into a reticular network after $4 \mathrm{~h}$. Therefore, we assume that the osmotic effect of R-hc in the dermis could be attributed to higher permeability.

HE staining is a classic method to detect the pathophysiological changes of skin, but it can only observe the skin's thickness changes in various layers, inflammatory cell invasion and other indicators, but the absorption \& distribution route and collagen content, which are directly related to wound healing or drug efficacy assessment, can not be observed. In our study, we examined SHG intensity to measure the content of collagen in the dermal skin. SHG imaging provides increased resolution and contrast when 
examining collagen than typical histological staining analysis [28]. Moreover, it is also a feasible and fast method that could be applied to the imaging of thicker tissue without fixing, slicing and staining [29]. SHG intensity could accurately reflect the in vivo changes in collagen. Specifically, in the examination of HE staining of R-hc, we found that the amount of R-hc gradually increased after iFluor-R$\mathrm{hc}$ administration and reached a peak at the $4 \mathrm{~h}$ time point. Similar results were also observed in the analysis of Franz-type diffusion, which was consistent with the findings of the in vivo SHG-TPEF skin imaging. These findings indicate that SHG imaging could provide reliable measurements for the real-time evaluation of collagen absorption.

Collagen is one of the core constituents that maintains the elasticity of skin; thus, reducing collagen in the skin would result in wrinkled and flabby skin [30]. In our study, we observed that R-hc gradually permeated the dermal skin and formed the reticular structure after $3 \mathrm{~h}$ of administration. This suggests that exogenous collagen could not only supplement skin collagen to meet the needs of anti-aging cosmetics but also form granulation tissue together with intrinsic collagen to fill wounds in tissue.

SHG-TPEF imaging allows us to observe the manner of transdermal absorption and the aggregation sites of R-hc in vivo, but some limitations still exist for this technology. For example, individual differences in cortical thickness might cause variation in the scanning results, which could have an impact on examining SHG intensity. Alternatively, the autofluorescence of tissue might affect the detection of exogenous collagen labeled with fluorescent probes. A previous study showed that the excitation wavelength of tissue autofluorescence was between $720 \mathrm{~nm}$ and $880 \mathrm{~nm}$, and the overall signal sensitivity (420-490 nm) was affected by the convolution of the spectral transmittance and the detector efficiency [31]. Therefore, the use of infrared dyes could be useful for the fluorescent labeling of exogenous proteins, and bandpass filters could be adjusted to reduce the effects of autofluorescence on skin scanning.

\section{Conclusion}

In this study, we used in vivo SHG-TPEF skin imaging to track the dynamic process of the transdermal absorption of exogenous collagen in a mouse model. We observed that exogenous collagen gradually permeated the dermal skin and formed a reticular structure, which was also confirmed by traditional in vitro skin imaging. This is the first study to use a noninvasive approach to visualize the absorption of exogenous collagen in skin in vivo, which could extend our understanding of the process of skin absorption and provide the feasible technology for the clinical assessment of drugs for external use.

\section{Declarations}

Ethics approval and consent to participate:

The animal experiments were supervised and approved by the Research Ethics Committee of the China Academy of Chinese Medical Sciences( ERCCACMS21-2106-12). 
Consent for publication:

The work described has not been published before. It is not under consideration for publication elsewhere. Its publication has been approved by all co-authors and the responsible authorities at the institution where the work is carried out.

Availability of data and materials:

The datasets used or analysed during the current study are available from the corresponding author on reasonable request.

Competing interests:

The authors declare no conflicts of interest related to this article.

Funding:

This work was supported by the Fundamental Research Funds for the Central Public Welfare Research Institutes (JBGS2021007)『National Natural Science Foundation of Beijing, China (7192141) , National Key Research and Development Program of China (No. 2019YFC1709305) and National Natural Science Foundation of China (81603285).

Authors' contributions:

Conception and design $\triangle$ Yi Wang. Drafting the manuscript: Yanan Sun. Operating the two-photon fluorescence microscope: Yanan Sun. 3D image analysis: Shuhua Ma; Franz-type diffusion: Gaiying He; Immunofluorescence\&HE staining: Weifeng Yang

Acknowledgements:

The authors would like to thank TianJin Irheaya Biological Technology Co., Ltd. for providing R-hc.

\section{References}

1. Bouwstra JA, Honeywell-Nguyen PL, Gooris GS, et al. Structure of the skin barrier and its modulation by vesicular formulations. Progress in lipid research. 2003;42(1):1-36.

2. Menon GK. Skin basics; structure and function. Lipids and Skin Health: Springer 2015:9-23.

3. Ngan CL, Basri M, Tripathy M, et al. Skin intervention of fullerene-integrated nanoemulsion in structural and collagen regeneration against skin aging. European Journal of Pharmaceutical Sciences. 2015;70:22-28.

4. Di Lullo GA, Sweeney SM, Körkkö J, et al. Mapping the ligand-binding sites and diseaseassociated mutations on the most abundant protein in the human, type I collagen. Journal of Biological 
Chemistry. 2002;277(6):4223-4231.

5. Sun W, Inayathullah M, Manoukian MA, et al. Transdermal delivery of functional collagen via polyvinylpyrrolidone microneedles. Annals of biomedical engineering. 2015;43(12):2978-2990.

6. Toki F, Honkura N, Shirakata Y, et al. Second harmonic generation reveals collagen fibril remodeling in fibroblast-populated collagen gels. Cell structure and function. 2013:13017.

7. Chai H-J, Li J-H, Huang H-N, et al. Effects of sizes and conformations of fish-scale collagen peptides on facial skin qualities and transdermal penetration efficiency. Journal of BioMed Research. 2010;2010.

8. Fediuk DJ, Wang T, Chen $\mathrm{Y}$, et al. Metabolic disposition of the insect repellent DEET and the sunscreen oxybenzone following intravenous and skin administration in rats. International journal of toxicology. 2012;31(5):467-476.

9. Schulz J, Hohenberg H, Pflücker F, et al. Distribution of sunscreens on skin. Advanced drug delivery reviews. 2002;54:S157-S163.

10. Cross SE, Innes B, Roberts MS, et al. Human skin penetration of sunscreen nanoparticles: in-vitro assessment of a novel micronized zinc oxide formulation. Skin pharmacology and physiology. 2007;20(3):148-154.

11. Gamer A, Leibold Ev, Van Ravenzwaay B. The in vitro absorption of microfine zinc oxide and titanium dioxide through porcine skin. Toxicology in vitro. 2006;20(3):301-307.

12. Denk W, Strickler JH, Webb WW. Two-photon laser scanning fluorescence microscopy. Science. 1990;248(4951):73-76.

13. König K, Ehlers A, Riemann I, et al. Clinical two-photon microendoscopy. Microscopy research and technique. 2007;70(5):398-402.

14. Campagnola PJ, Loew LM. Second-harmonic imaging microscopy for visualizing biomolecular arrays in cells, tissues and organisms. Nature biotechnology. 2003;21(11):1356.

15. Pittet J-C, Freis $\mathrm{O}$, Vazquez-Duchêne M-D, et al. Evaluation of elastin/collagen content in human dermis in-vivo by multiphoton tomography-variation with depth and correlation with aging. Cosmetics. 2014;1(3):211-221.

16. Thrasivoulou C, Virich G, Krenacs T, et al. Optical delineation of human malignant melanoma using second harmonic imaging of collagen. Biomedical optics express. 2011;2(5):1282-1295.

17. Kiyomatsu H, Oshima Y, Saitou T, et al. Quantitative SHG imaging in osteoarthritis model mice, implying a diagnostic application. Biomedical optics express. 2015;6(2):405-420. 
18. Miller DR, Jarrett JW, Hassan AM, et al. Deep tissue imaging with multiphoton fluorescence microscopy. Current opinion in biomedical engineering. 2017;4:32-39.

19. Ma S, Sun Y, Yang W, et al. Repair effect and mechanism of recombinant human collagen on laser induced damage of mice skin. Acta laser biology sinica. 2018;27(5):399-406.

20. Dunn AK, Wallace VP, Coleno M, et al. Influence of optical properties on two-photon fluorescence imaging in turbid samples. Applied optics. 2000;39(7):1194-1201.

21. Theer P, Denk W, Sheves $M$, et al. Second-harmonic generation imaging of membrane potential with retinal analogues. Biophysical journal. 2011;100(1):232-242.

22. Li C, Wang J, Sun Y, et al. Comparison of the stability and biological activity of two fluorescent labeling Pilose antler extracts. Acta laser biology sinica 2019;28(1):46-53.

23. Sun Y, Zhao J, Li C, et al. Application of second harmonic generation and two-photon fluorescence in the distribution tracing of fluorescence labelled collagen. Acta laser biology sinica. 2017;26(1):24-29.

24. Ogiso $\mathrm{T}$, Iwaki $\mathrm{M}$, Tanino $\mathrm{T}$, et al. In vitro skin penetration and degradation of peptides and their analysis using a kinetic model. Biological and Pharmaceutical Bulletin. 2000;23(11):1346-1351.

25. Elias PM, Choi EH. Interactions among stratum corneum defensive functions. Experimental dermatology. 2005;14(10):719-726.

26. Potts RO, Guy RH. Predicting skin permeability. Pharmaceutical research. 1992;9(5):663-669.

27. Lin R-Y, Hsu C-W, Chen W-Y. A method to predict the transdermal permeability of amino acids and dipeptides through porcine skin. Journal of Controlled Release. 1996;38(2-3):229-234.

28. Tanaka R, Fukushima S-i, Sasaki K, et al. In vivo visualization of dermal collagen fiber in skin burn by collagen-sensitive second-harmonic-generation microscopy. Journal of biomedical optics. 2013;18(6):061231.

29. Sun $\mathrm{C}-\mathrm{K}$, Chen $\mathrm{C}-\mathrm{C}$, Chu S-W, et al. Multiharmonic-generation biopsy of skin. Optics letters. 2003;28(24):2488-2490.

30. Lee $\mathrm{CH}$, Singla A, Lee Y. Biomedical applications of collagen. International journal of pharmaceutics. 2001;221(1-2):1-22.

31. Breunig HG, Studier H, König K. Multiphoton excitation characteristics of cellular fluorophores of human skin in vivo. Optics express. 2010;18(8):7857-7871.

\section{Figures}



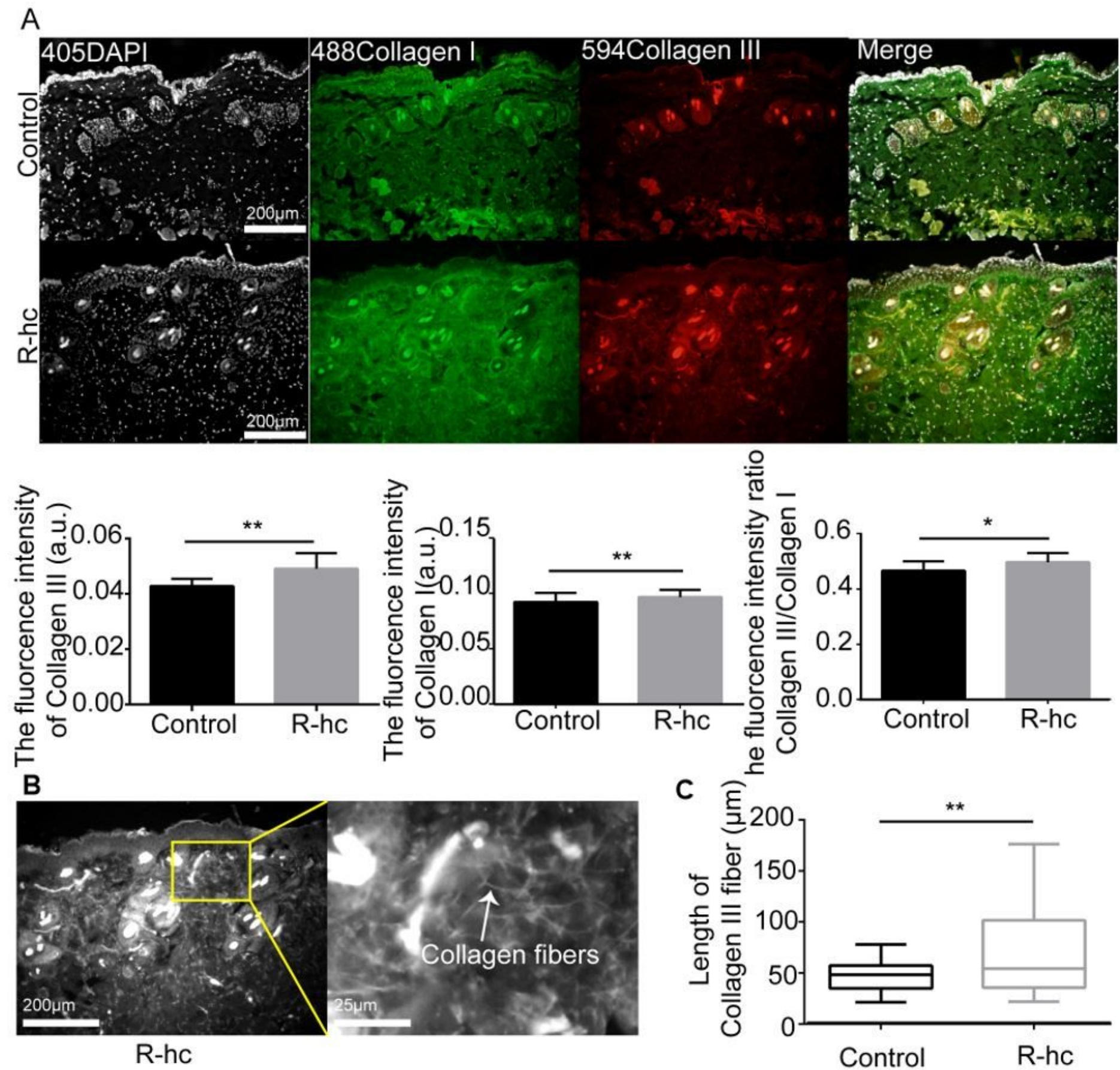

Figure 1

R-hc could be transdermally absorbed by skin. (A) Immunofluorescence results of R-hc absorption (Above panel). Statistical analysis of Type III and Type I collagen fluorescence intensity (Below panel) and the ratio of type III and type I collagen. (B) Type III collagen in the dermis were mutually linked into reticular structures. (C) Fiber length analysis of Type III collagen. ${ }^{*} p<0.05,{ }^{*} p<0.01$. 

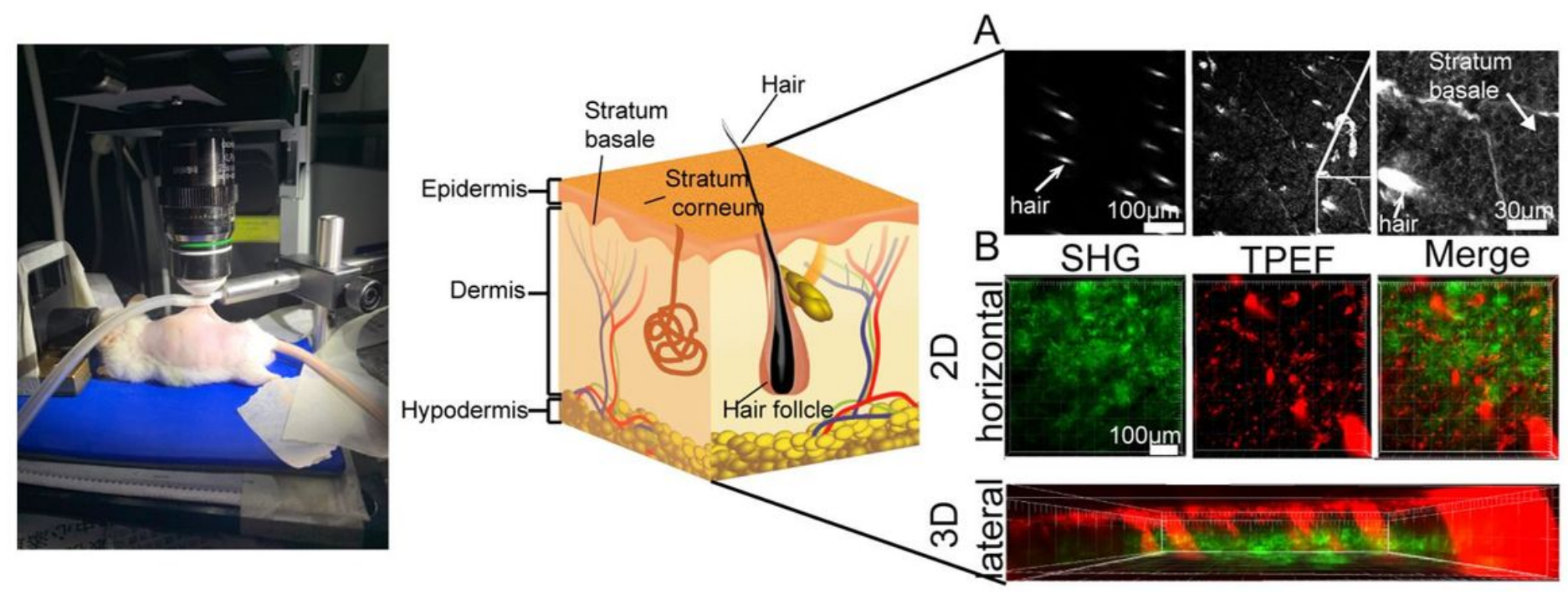

\section{Figure 2}

Schematic illustration of skin imaging. Left panel: in vivo SHG-TPEF skin imaging platform. Middle panel: schematic diagram of the skin structure. Right panel: scanning images of different layers of the skin, including epidermis skin (A), dermis skin in 2D and 3D images (B). 

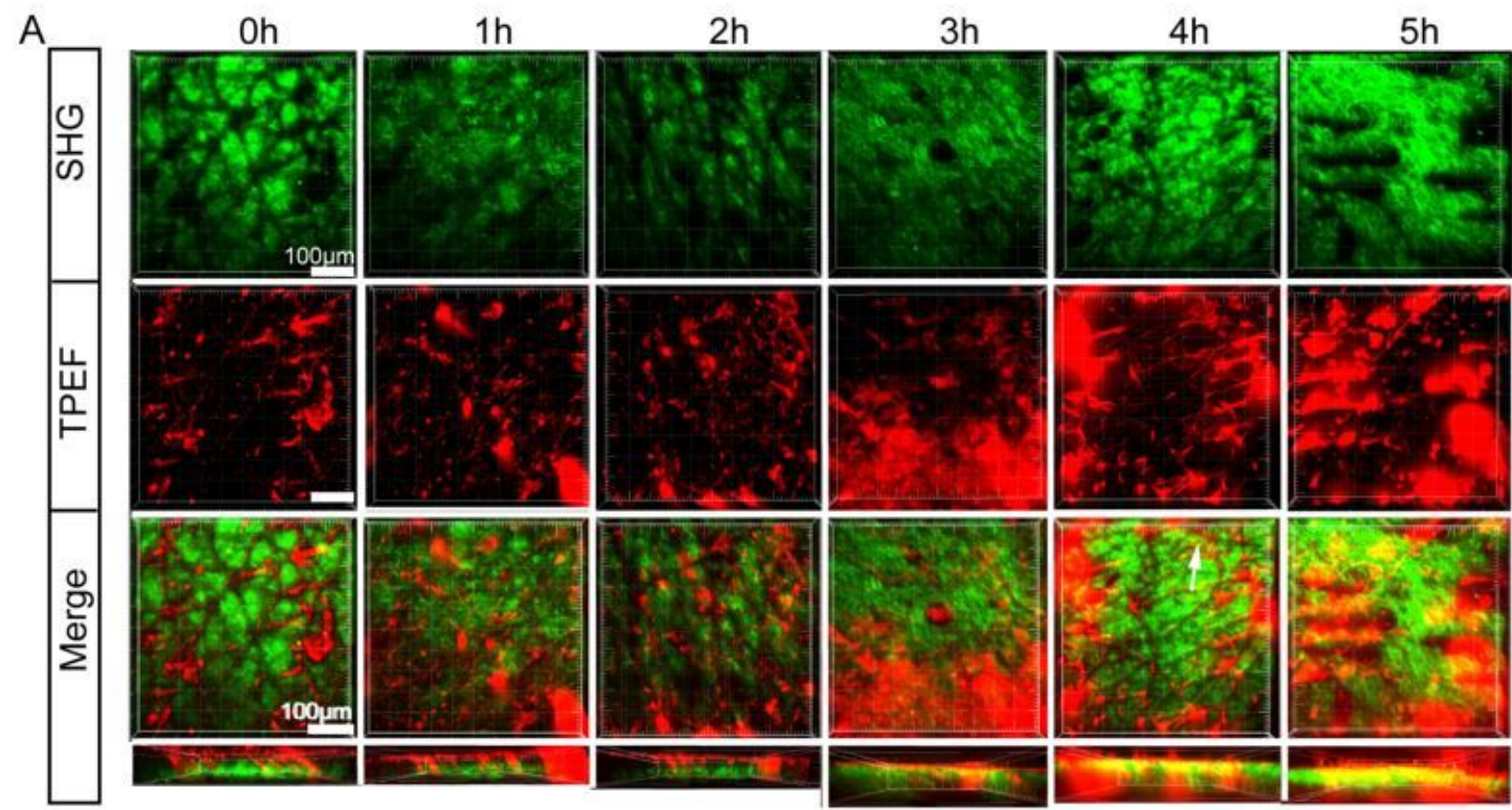

B

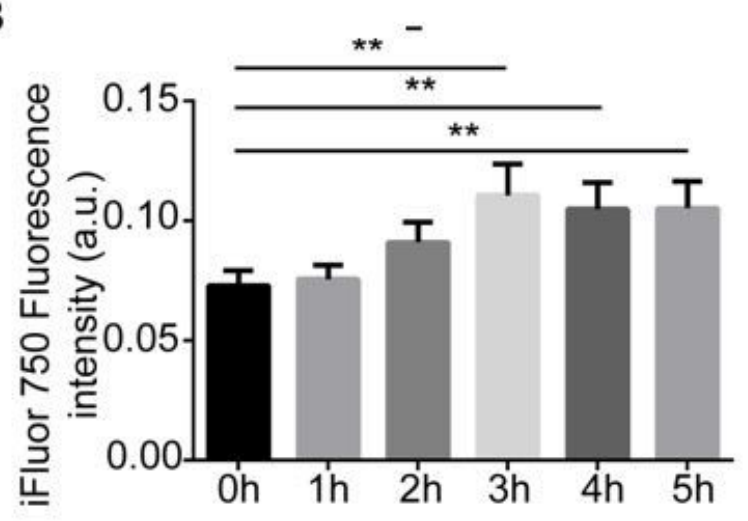

C

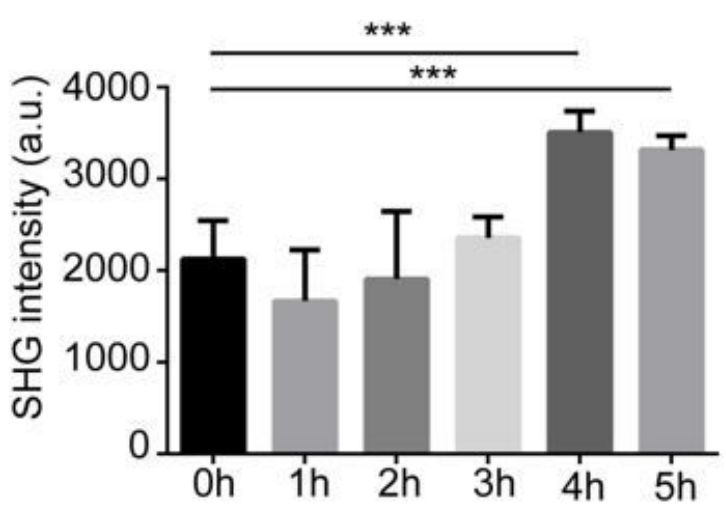

\section{$D$}

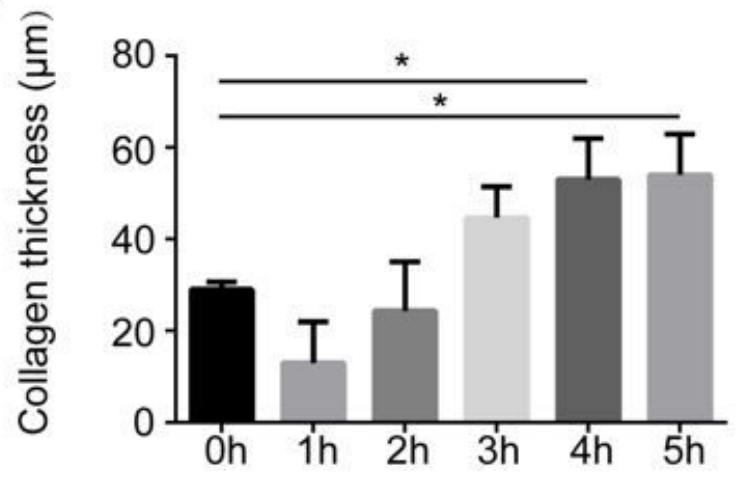

Figure 3

High-resolution images illustrated the process of iFluor-R-hc absorption by in vivo SHG-TPEF imaging. (A) SHG images and TPEF images of iFluor-R-hc. White arrow represents reticular structure. (B) Statistical analysis of iFluor 750nm intensity. (C) Statistical analysis of maximum SHG intensity. (D) Statistical analysis of collagen layer thickness. Each time points showed the mean value and standard deviation of samples. ${ }^{\star} p<0.05,{ }^{\star \star} p<0.01,{ }^{\star \star *} \mathrm{p}<0.001$. 


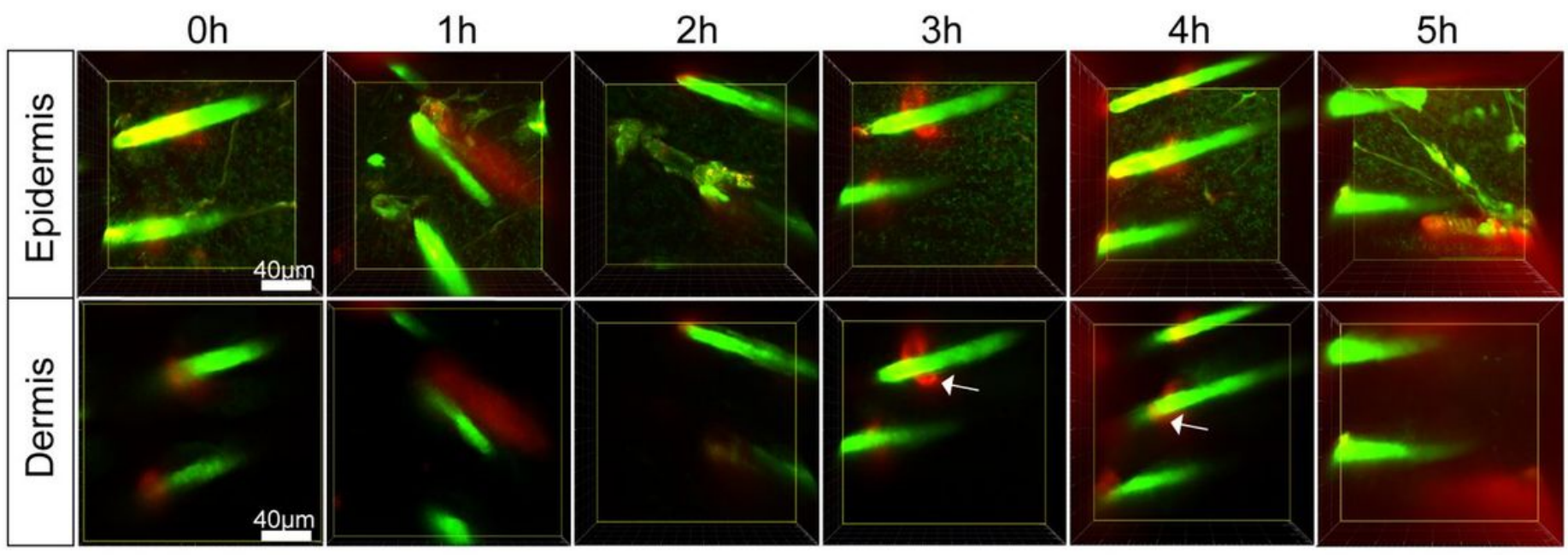

Figure 4

SHG-TPEF imaging of iFluor-R-hc around hair follicle. Green: hair shaft and stratum spinosum/stratum basale $(\lambda=750 \mathrm{~nm})$; Red: iFluor-R-hc $(\lambda=790 \mathrm{~nm})$; White arrow: iFluor-R-hc in hair follicle. 


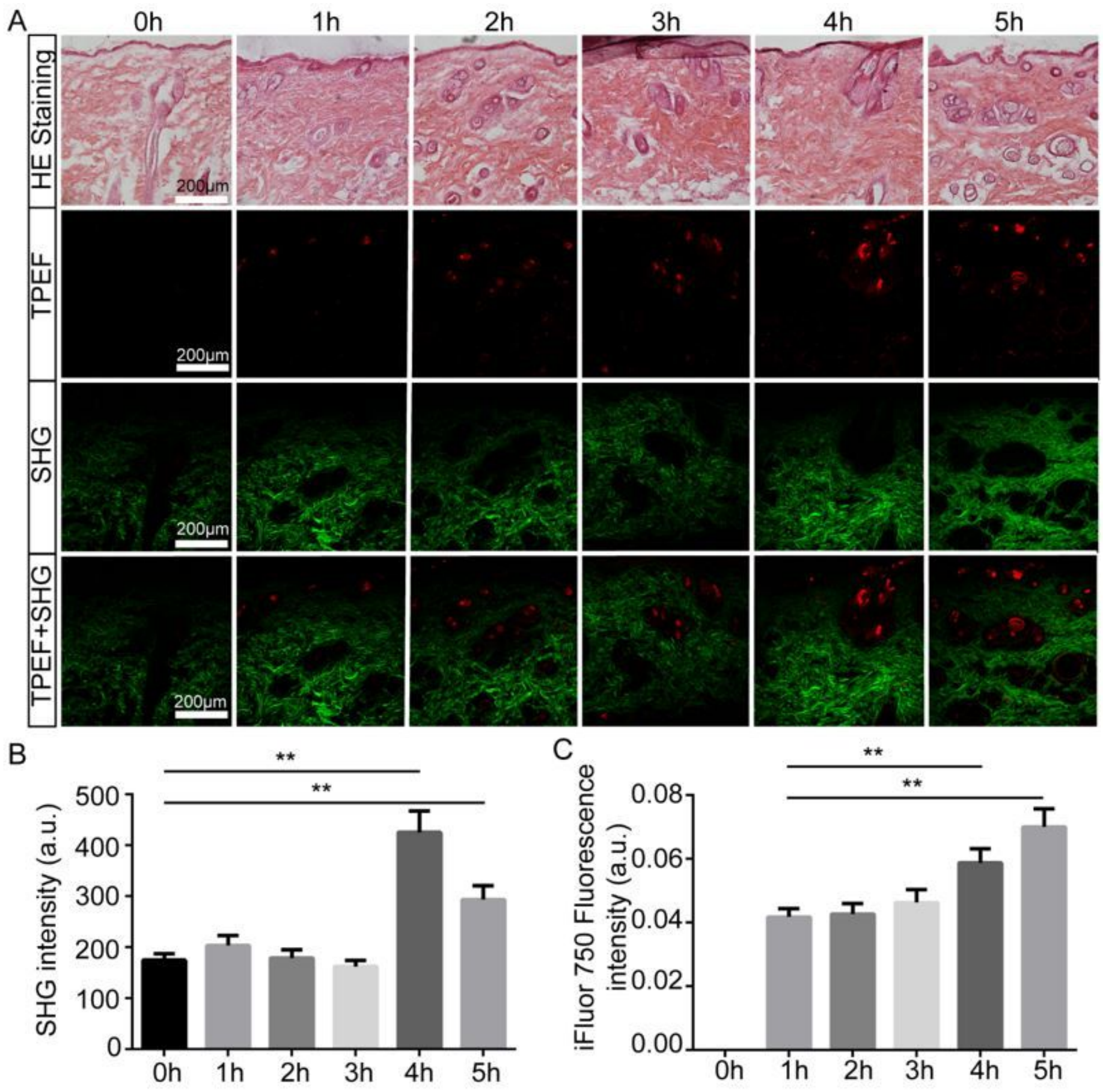

Figure 5

Histological examination of skin after iFluor-R-hc administration. (A) HE and SHG-TPEF imaging of skin after iFluor-R-hc administration. (B) Statistical analysis of SHG intensity. (C) Statistical analysis of iFluor intensity. ${ }^{\star *} \mathrm{p}<0.01$. 


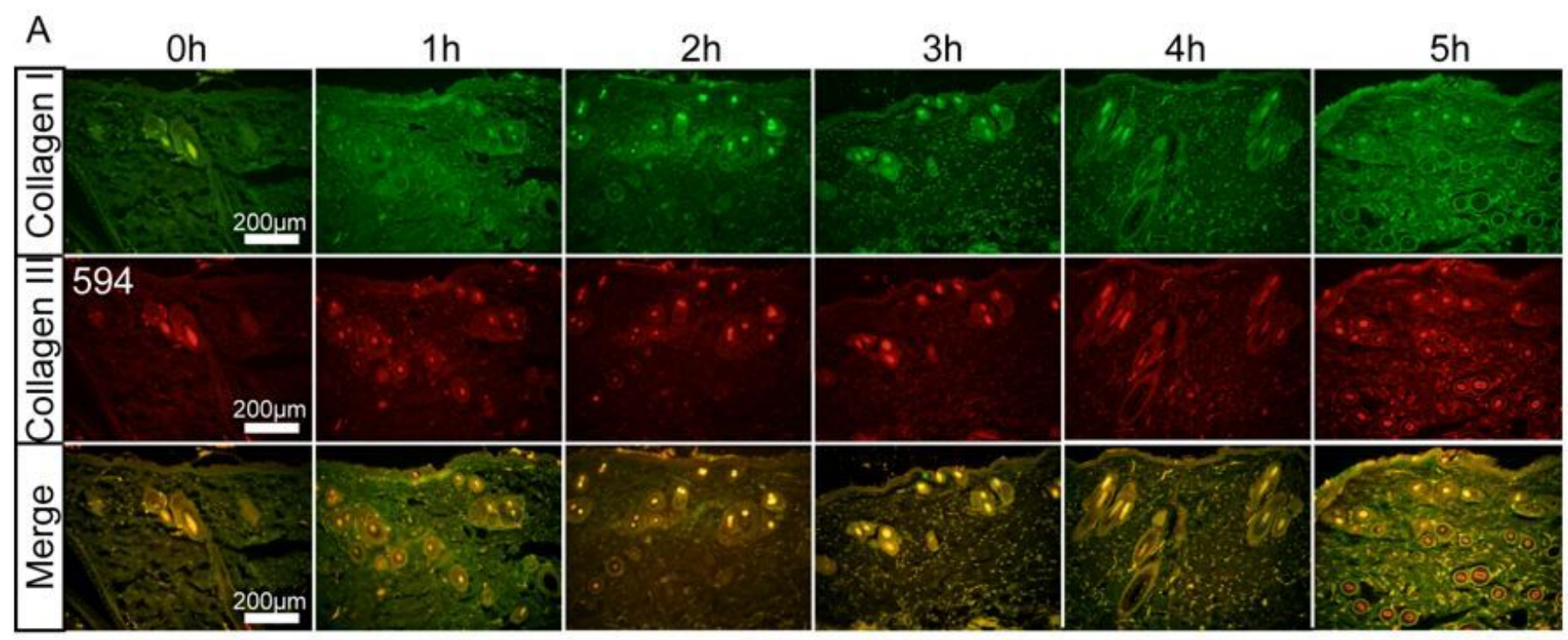

B
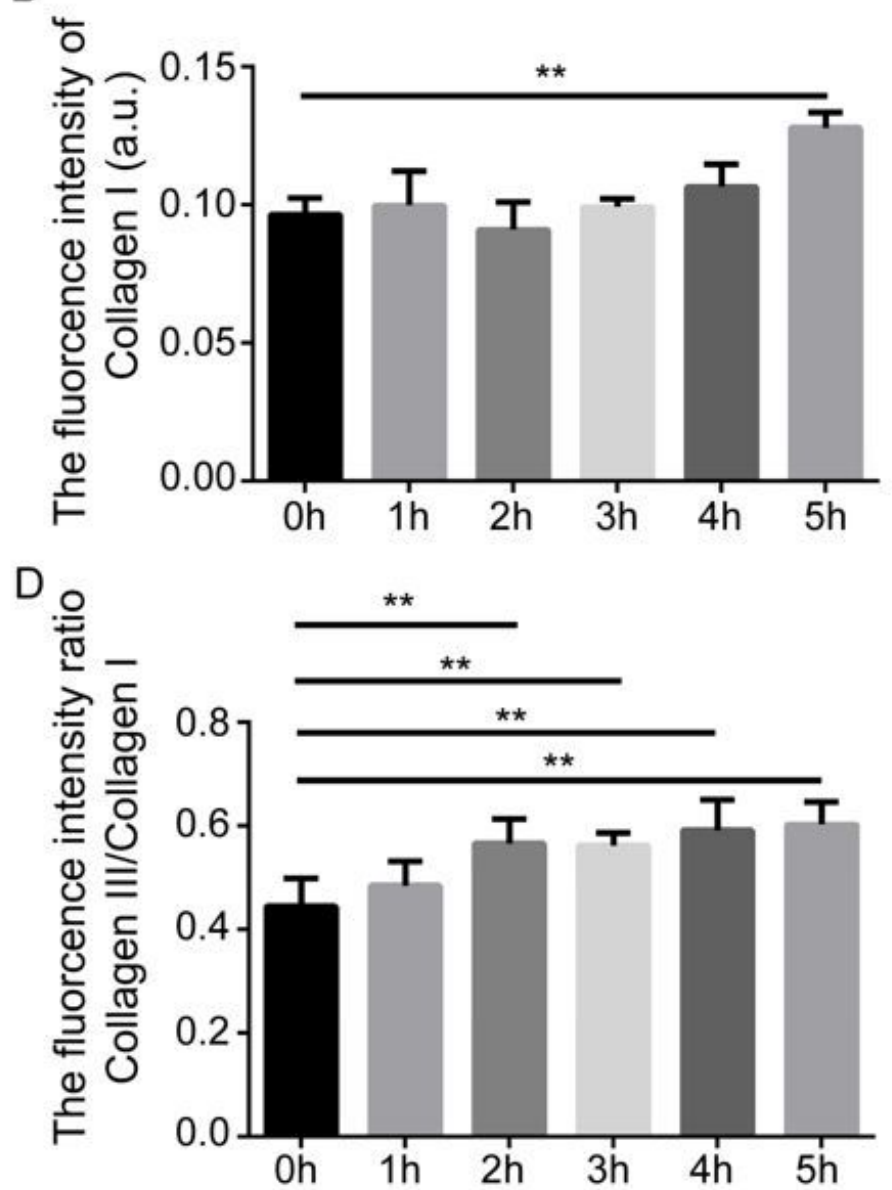

C

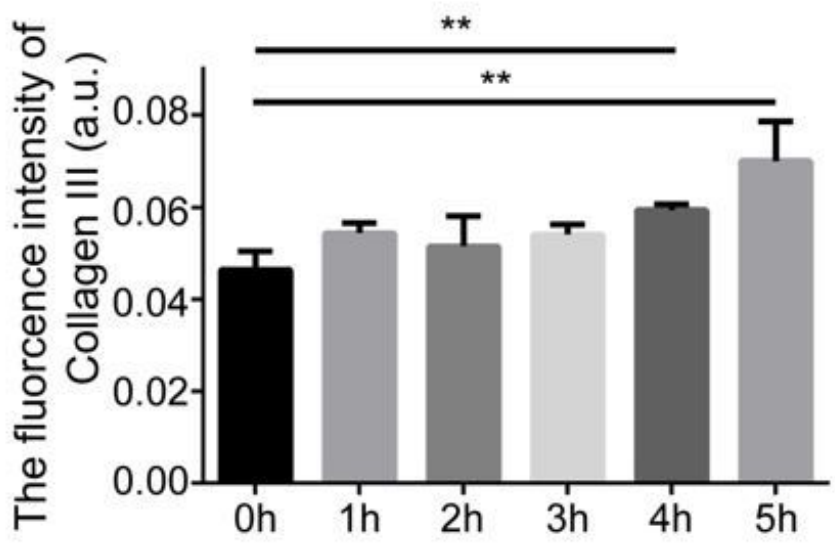

Figure 6

Quantification of collagen. (A) Immunofluorescence imaging of R-hc absorption. (B-D) Statistical analysis of Type III and Type I collagen fluorescence intensity and the ratio of type III and type I collagen. ${ }^{*} \mathrm{p}<0.01$. 

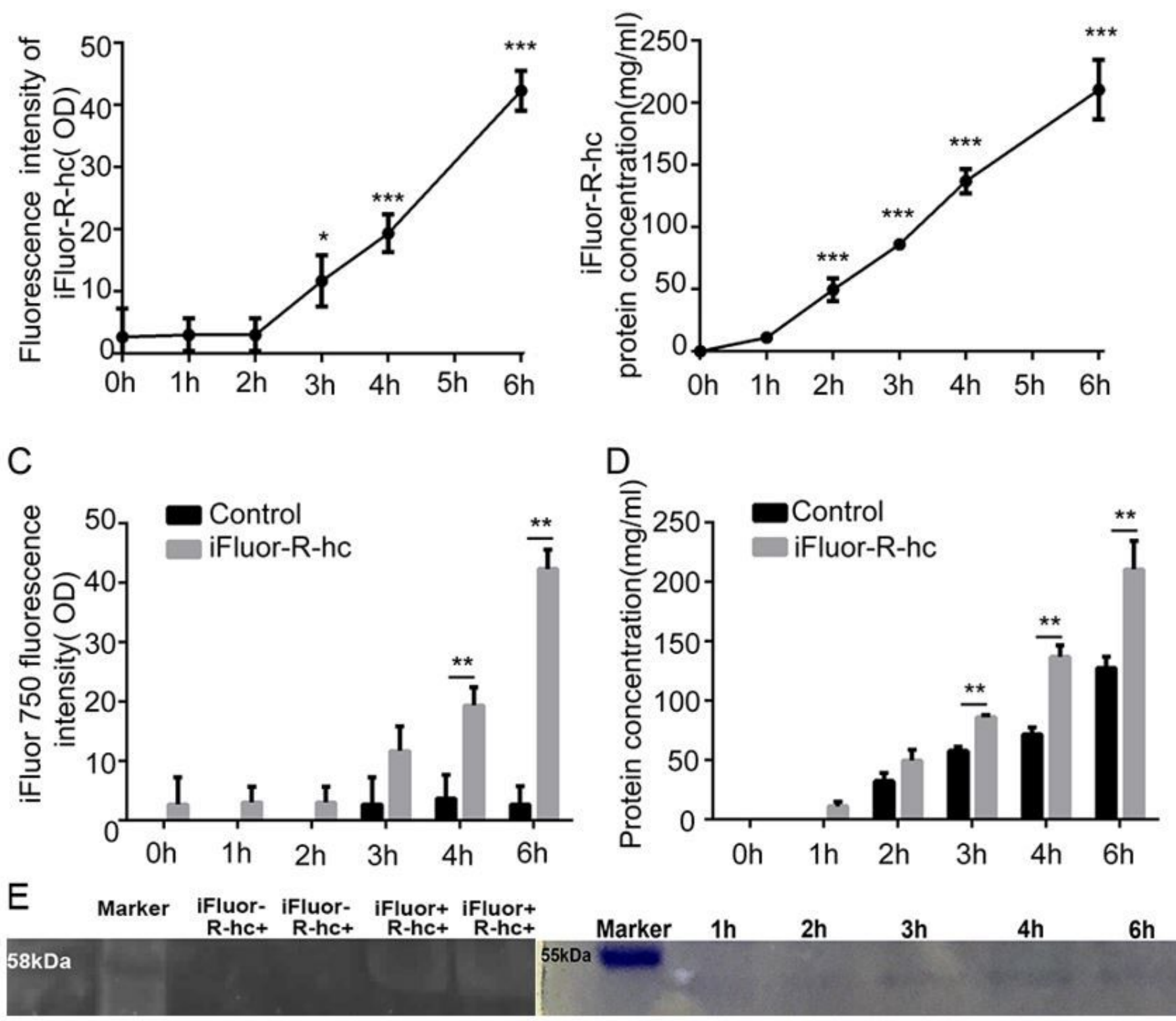

\section{Figure 7}

In vitro examination of iFluor-R-hc Transdermal absorption. (A) iFluor-R-hc fluorescence intensity detected by fluorescence microplate reader. (B) Determination of iFluor-R-hc concentration by BCA method. (C) iFluor-R-hc fluorescence intensity compare to control group (normal saline). (D) iFluor-R-hc concentration compare to control group (normal saline). (E)R-hc was labeled with iFluorTM 750 SE(Left pane),Ex=700nm . Coomassie blue staining of iFluor-R-hc (Right pane). ${ }^{\star \star} p<0.01$.

\section{Supplementary Files}

This is a list of supplementary files associated with this preprint. Click to download.

- graphicsabstract.jpg 